\title{
The CRISPR/Cas9 system in gene editing of cancer stem cells
}

\author{
By Manreet (Sonia) Padwal
}

It has been well documented that cancer development is a result of multiple mutations within tumor cells. Unfortunately, current treatments do not guarantee complete removal of the tumor and the cancer may reappear. Much existing literature suggests that a small population of cells within the tumor has the ability to induce tumor formation and growth (1). This small population of cells, referred to as cancer stem cells, seem to arise from the accumulation of mutations in normal stem cells over a long period of time. Opposing theories still suggest all cells have the ability to initiate tumorigenesis (Branavan Manoranjan. Conversation with Manreet Padwal. 2016 Dec 15*), but the stem cell theory has been well established in leukemia and solid malignancies (1).

With accumulating evidence in support of the cancer stem cell theory, scientists are investigating an approach to specifically target the tumor-initiating stem cell (1). Current therapies are centered around treating the bulk of tumor cells and fail to treat the tumor-initiating stem cell population. If any of the stem cells survive, the cancer may reoccur (1), which is why emphasis has shifted to direct targeting of the tumor stem cell population. Dr. Sheila Singh of McMaster University is leading research in investigating the most frequent form of brain cancer: glioblastoma. Her research focuses on identifying tumor stem cell markers and generating a customized approach to target and eliminate these specific cells. Branavan Manoranjan, an MD/PhD student in Dr. Singh's lab, notes that “markers that identify stem cells in the brain have not been concretely identified and this ultimately comes down to methodology."

Gene editing technology is a powerful tool applicable to genome editing in a range of pathologies (2). Investigators are beginning to use a more simple and versatile CRISPR/Cas9 gene editing system to identify new cancer stem cell markers. Dr. Chitra Venugopal, a Research Associate in Dr. Sheila Singh's lab, is using CRISPR/Cas9 to map out the tumor landscape in human glioblastoma by altering genes to understand their role in tumor formation. "With the ease of CRISPR/Cas9, everyone is transitioning" (Dr. Chitra Venugopal. Conversation with
Manreet Padwal. 2016 Dec 15*). According to Manoranjan, "a study like this gives us an idea of what genes are expressed at that particular time point and therefore we can create a customized therapy for that target."

Current investigators have identified the expression of CD133 as a marker of cancer stem cells and a potential therapeutic target (3). Dr. Venugopal is trying to develop a more selective approach to identify CD133-positive cells and is using a drug or antibody to target these cells. Researchers are also investigating the possibility of using the patient's immune cells to target cancer antigens. To this end, the CRISPR/ Cas 9 system can be used to edit the immune cell genome to better recognize antigens on the tumor (4). Dr. Venugopal discusses the potential of using immune cells to recognize antigens such as CD133 as a means of specifically targeting tumor-initiating stem cells. "The ultimate goal would be to design an immune cell to hit multiple antigens at the surface," says Manoranjan.

A Chinese team is already using the CRISPR/Cas9 gene editing technique to engineer immune cells to knock out PD-1, a gene cancer cells take advantage of that acts to dampen the immune response, ultimately resulting in less detection of cancer cells. Thus, deletion of PD-1 would create more active immune cells and boost the immune system in fighting cancer more effectively (5). Although scientists are still far from perfecting this treatment, the CRISPR/Cas9 gene editing technique is making headway and shows promise in the field of cancer research. With further investigation, CRISPR technology will allow scientists to modify the immune system to directly target cancer stem cells, potentially leading to a strategy to treat and prevent the progression of cancer.

\section{References}

1. Yoo M, Hatfield D. The cancer stem cell theory: Is it correct? Mol Cells. 2008; 26(5), 514-516.

2. Joung J, Sander Jeffry. TALENs: a widely applicable technology for targeted genome editing. Nat Rev Mol Cell Biol. 2013I 14(10), 49-55.

3. Ferrandina G, Petrillo M, Bonanno G, Scambia G. Targeting CD133 


\section{HeAlth SCIENCE InQuiry}

4. Osborn J, Webber B, Knipping F, Lonetree C, Tennis N, DeFeo A, McElroy A, Starker C, Lee C, Merkel S, Lund T, Kelly-Spratt K, Jensen M, Voytas D, Von Kalle C, Schmidt M, Gabriel R, Hippen K, Miller J, Scharenberg A, Tolar J, Blazar B. Evaluation of TCR Gene editing achieved by TALENS, CRISPR/Cas9 and megaTAL Nucleases. Mol Ther. 2016; 24(3), 570-581.

5. Cyranoski D. Chinese scientists to pioneer first human CRISPR trial. Nature. 2016; 535, 476-477.

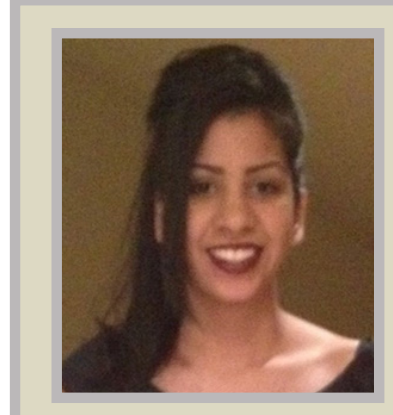

Manreet (Sonia) Padwal

Sonia is a PhD candidate at McMaster University with a BSC in Life Sciences. Her doctoral research focuses on elucidating the role of matrix metalloproteinases and WNT signalling in the development of peritoneal membrane injury. Sonia is also involved in clinical research where her primary objective involves understanding how to better protect and preserve the peritoneal membrane for patients on peritoneal dialysis. She is also passionate about the visual arts and has submitted her work to local galleries and events. 